Editorial

\title{
Question-response sequences in conversation across ten languages: An introduction
}

Questions have played a central role in thought about language. In the philosophy of language, the contrast with declaratives has been a lever with which to examine theories of the match between language and the world, a way of exploring presupposition and information structure, and a route into the theory of speech acts. In anthropology, the special social role of questions has long been noted, on the one hand displaying the ignorance appropriate to lower status, on the other hand the power of coercion (Goody, 1978). In grammar, interrogatives are often marked sentence types, and the operations converting declaratives to interrogatives have proved to be an enlightening window on the underlying syntactic machinery of language in general. Questions have also been held to be a crucial locus where intonation, grammar and function coalesce. In pragmatics, the tie between question and answer has been a prototype for larger units in the structure of verbal interaction, and specifically in the theory of adjacency pairs.

In this special issue, the focus is on the pragmatics of questions and their responses. As the ethnographers of speaking have pointed out (Hymes, 1962), many activities are partially or wholly constituted by question-answer sequences, such as the news interview (Clayman and Heritage, 2002), the press conference (Clayman et al., 2006, 2007), and the criminal trial (Atkinson and Drew, 1979). While substantial research has been devoted to the asking of questions in institutional encounters from the classroom (e.g., Mehan, 1985) to the consulting room (e.g., Boyd and Heritage, 2006), in comparison, there has been relatively less research about questions and responses in informal conversation (but see for instance Freed, 1994; Steensig and Drew, 2008; Weber, 1993). Yet in everyday social interaction, questions are ubiquitous, and as long noted in the 'indirect speech act' literature, questions are often the vehicle for other actions, from repair (What did you say?), to offers (Would you like a hand?), requests (Do you have a spare pencil?), challenges (Why are you doing that?), and so on, further increasing their prominence in the conduct of conversation. Further, as the prototypes for adjacency pairs, question-response patterns offer special insights into the 'glue' that holds human interaction together-the patterns of expectation, the modulations possible in the wide range of available response types (non-answers/answers, partial/whole answers, direct/indirect answers, etc.) and response formats (partial repetitions, response tokens of various kinds from nods to yes, one word answers, etc.).

For all these reasons, questions and their responses recommend themselves as a crucial locus for research. But if the literature on questions in everyday interaction is sparse, almost totally absent are systematic cross-linguistic surveys of the use of questions in such contexts. This special issue reports on the first effort (as far as we know) to conduct a survey of this sort. We were interested in how interactants design and use questions in everyday social interaction across typologically diverse languages: What special affordances do the different modes of coding for interrogativity offer? How are questions recognized when the language provides no means for morphosyntactic coding (as for polar questions in Italian or Yélî Dnye)? What if the question-coding falls at the end of the turn, where it is late perceived and vulnerable to overlap? Do differences in temporal patterning of responses reflect such coding differences? In addition, we were interested in the patterning of responses. Question-answering systems have been said to differ in type (see e.g., Jones, 1999): for example, in polar questions, some answering systems are said to be polarity based vs. proposition based (A: "Isn't he coming?” B: “No (he's not)" vs. "Yes (you're right he isn't coming)"). Equally, some languages are said to sparsely use interjections such as 'yes' or 'no' in response, but to insist on repetitional answers ("He isn't coming"). What consequences for the timing and conduct of interaction do such differences have?

To address these kinds of questions, we formed a team of ten researchers, each working on a different language, allowing us to draw extensive samples of natural conversation from five continents to systematically compare strategies for asking and responding to questions in naturally occurring spontaneous conversation.

\section{Our guiding questions}

Underlying the specific questions that we can ask of the data, the fundamental question that drives this work concerns the search for universals in human behaviour. Do people in different linguistic communities adopt different interactional patterns, following the specific affordances of their languages or culturally specific behavioural norms? There is a 
widespread view that language structure is universal in its essence while patterns of language use are free to vary according to cultural influence (as spelled out in Hymes, 1962). The reverse assumption is at least as plausible: languages themselves are highly culturally variable (Evans and Levinson, 2009), while "the uncodified, unnoticed, low-level background of usage principles or strategies may be fundamentally culture-independent ... because they are the prerequisites for the system to work at all" (Levinson, 2000:xiv; see also Enfield and Levinson, 2006). The question-response sequence provides an excellent site for investigation of this general question because it is (as far as we know) a universal unit of conversational organization, and a pervasive type of sequence in all communities.

Within the general domain of question-response sequences we are interested in five core issues raised by prior research: (1) the formal coding of questions, (2) the social actions performed by questions, (3) normative preferences for responding to questions (e.g., relating to speech participant roles like addressee vs. bystander), (4) relative preferences for types of answer (e.g., interjection versus repetitional strategies), and (5) the role of visible behaviour (e.g., nodding, eye gaze). We say something about each of these in the following paragraphs.

\subsection{Formal coding of questions}

We wanted to examine the extent to which speakers rely on the formal encoding of questions (in lexicon, morpho-syntax or prosody) as opposed to working from the pragmatics of the question's content, eye gaze, or other cues. This was in part because we knew that some languages do not mark polar questions morphosyntactically or lexically, and in part because even when they can do so, questions are sometimes produced without such marking. For example, speakers often make ostensible assertions about their interlocutor ("B") which are interpreted as queries that person B will regularly confirm or disconfirm (e.g., "You have a younger brother" - "I do") (Heritage and Roth, 1995; Labov and Fanshel, 1977). Prosodic marking alone is unlikely to be entirely unambiguous, and has in fact in English been shown to occur less than half the time in declarative questions (Couper-Kuhlen, in press; Geluykens, 1988).

\subsection{Social action of questions}

Guided by the speech act and conversation analytic literature on the importance of considering what an utterance is "doing" in social interaction (Levinson, 1983; Searle, 1969), we sought to document the range of social actions that speakers use questions to implement, from information-seeking through to repair initiation (Schegloff et al., 1977), from requesting (Curl and Drew, 2008) to assessing (Heritage and Raymond, 2005), and the effect of this on the delivery of the response (Schegloff, 1968).

\subsection{Normative preferences for responding to questions}

In English there is a strong normative order surrounding questions. In the first place, responses are normatively required (Schegloff, 1968), and answers are preferred over non-answer responses such as “I don't know" or "I can't remember”(Clayman, 2002; Stivers and Robinson, 2006) in the technical sense that answers tend to be unmarked, while non-answers tend to be delayed, accompanied by explanations and the like (Levinson, 1983:307ff). Secondly, speakers commonly select individuals to respond to questions (Sacks et al., 1974), and selected question recipients have greater rights to respond than non-selected question recipients (Stivers and Robinson, 2006). We asked whether we would see these same orientations in a variety of languages.

\subsection{Relative preference for types of answer}

If the preferred response to a question is an answer, preference organization operates once again at the level of which kind of answer is preferably deployed. Two preferences in answer design for polar questions have been documented for English. First is a preference for agreement (Heritage, 1984; Pomerantz, 1984; Sacks, 1987). Answers that are preferred by the question in structural terms (e.g., a confirmation as a response to a non-negative polar interrogative; A: Has John left? B: Yeah) are delivered more quickly, without mitigation and without accounts. We asked whether this would be true across other languages. A second preference in English is that full form responses that repeat all or part of the question as a method of confirming or (with negation) disconfirming (e.g., A: Has John left? B: He's left), are structurally "nonconforming" relative to the question (Raymond, 2003). They also assert relatively more agency or authority over the proposition than interjection responses such as "Yeah" (Heritage and Raymond, in press; Stivers and Hayashi, 2010). The linguistics literature suggests that many languages normally or even prescriptively use repetition instead of interjections like yes/no. Is it really the case, we wondered, that the pragmatically unmarked answer might be an interjection type answer (yes/no) in one language but a repetitional type in another, when both were available?

\subsection{The role of visible behaviour}

Relatively little is known about the use of visible responses to questions such as head nods and shakes, extended eye blinks or points (but see Kendon, 2002; Kita and Ide, 2007; Maynard, 1987 on head movements, though not specific to the 
Table 1

\begin{tabular}{lll} 
Languages included in the project. & & \\
\hline Language name & Language group & Data collected by \\
\hline fäkhoe Haillom (Namibia) & Khoisan & Hoymann \\
Danish & Germanic & Englemem \\
Dutch & Germanic & Stivers \\
English (US) & Germanic & Rossano \\
Italian & Romance & Hayashi \\
Japanese & Isolate & Yoon \\
Korean & Ural-Altaic & Enfield \\
Lao & Tai & Brown \\
Tzeltal (Mexico) & Mayan & Levinson \\
Yélî Dnye (Papua New Guinea) & Isolate & \\
\hline
\end{tabular}

question-response sequential environment). In the context of extended tellings, Levinson and Brown (2004), relying on qualitative analyses of two languages, Yélî Dnye and Tzeltal, show that Yélî Dnye speakers rely heavily on visible behaviour to acknowledge their interlocutors. By contrast, Tzeltal speakers rely more heavily on extended cycles of repetition (Brown, 1998) relying relatively little on visible behaviour. We have found that these differences are associated with quantitative differences in gaze behaviour in the question-response context (Rossano et al., 2009). We wanted to document the extent to which people rely on visible responses in different cultures.

\section{Analytic methods}

Earlier studies of questioning have often been qualitative, and where quantitative (Geluykens, 1988; Stenström, 1984) have been confined to a single language and not focused on the link between question and answer. Jakob Steensig and Paul Drew's edited special issue of Discourse Studies entitled "Questioning" (Steensig and Drew, 2008) was both cross-linguistic and attentive to the tie between question and response, but it provided no precise methods of comparison and was not quantitative. In contrast, our aims were explicitly comparative and we therefore adopted a quantitative approach from the outset, which would give us clear parameters of comparison.

Quantitative analysis relying on behaviour coding a la Bales (1950) has been much criticized for losing all the rich qualitative texture of verbal interaction (Stiles, 1989; Tuckett et al., 1985). This is a valid enough complaint when the categories counted are imposed by fiat. But there is another approach, namely to first do the qualitative work that demonstrates the kinds of categories that participants themselves use in the conduct of conversation, and then use these categories as the basis for quantitative analysis. This style of work has been successfully applied in, e.g., the study of doctorpatient interaction (Mangione-Smith et al., 2006; Stivers et al., 2003), and in examining changing styles of journalistic questioning of American presidents (Clayman et al., 2006, 2007).

In the studies reported here, behaviours are categorized, as far as possible, with reference to prior research on interaction, either in the target language itself, or in a number of other languages (typically including English). Using these data, we have recently been able to show remarkable similarities across languages in turn taking in conversation, for example in all the subtle factors affecting the timing of responses (Stivers et al., 2009).

Because our coding categories (see Stivers and Enfield, this issue) are based on existing qualitative analyses of interaction, we are confident that the categories are meaningful in interaction. Nevertheless, some behaviours were inevitably more straightforwardly codable than others. This was assessed during the pilot stage of the project where we refined the coding scheme, removing coding categories that contributors felt they could not reliably code. This sort of coding necessarily sacrifices detail, and cannot reflect all aspects of context, or capture the negotiability of moment by moment conversation. But the benefits of being able to systematically compare how speakers of ten languages behave in question-response sequences outweigh these costs for the purposes at hand. It is only by using methods of this sort that one can see that the practices in one language really stand out as unusual, or that other practices look like good candidates for universals of language use (Stivers et al., 2009).

\section{Data}

We aimed for a diverse sample of languages, but since participants in the project had to obtain substantial videotaped corpora of natural conversation, our sample was to some extent based on availability. Nevertheless we were able to carefully match data from 10 languages from 5 continents, from Europe, the USA, Southeast Asia, Mexico, Namibia, and Papua New Guinea. Table 1 gives an overview of contributors and languages.

It was important to make sure that our data from the different societies were as comparable as possible. For this reason, all contributors used only data from maximally informal social interaction in familiar settings between people who knew each other well. None of the data were institutional or staged. Because of significant cultural variation in terms of when conversation is least constrained, the specific activity context varied. Thus, for English and Italian conversations this is commonly but not exclusively during eating or drinking events; in contrast, Tzeltal and Yélî speakers hardly talk while 
eating. In $\neq \bar{A}$ khoe Haillom conversations, speakers were commonly stringing beads together or building wire figures. Tzeltal conversations often occurred without an additional activity going on simultaneously although the body and clothing and events in the environment are always available for attention shifting. All contributors had video recordings of these events that were used as primary data and were considered comparable.

For each language, contributors relied on a range of different speakers and interactions, as made available by local interactional norms (some conversations were dyadic, some multiparty, and all language samples came from multiple conversations (between 5 and 17 according to site). Generalizations could thus be made over a total of 101 conversations, and some hundreds of distinct speakers. The sampling of excerpts was designed so as to maximize diversity in the participants and to dilute individual or interaction-specific effects.

\section{The volume}

This special issue offers a descriptive overview of each of the 10 languages across each of the primary dimensions mentioned above. As mentioned earlier, we do not report full comparative results here. Some results have been reported already (Rossano et al., 2009; Stivers et al., 2009; Stivers and Hayashi, 2010) and others are currently in press (Stivers, forthcoming) or in preparation (Stivers et al., 2010). Nevertheless, in reporting the facts about each target language, the authors had access to quantitative comparative measures provided by the editors, allowing the vignette of each language to be positioned relative to all the others. The main goal of this special issue is thus to profile the general character of questionresponse sequences in each of the languages, and more specifically:

(1) to provide readers with an overview of the special linguistic resources in each language for asking and responding to questions, and to indicate how speakers of a particular language deploy those linguistic resources in question-response sequences in everyday conversation, and

(2) to deliver a baseline for comparing questioning across languages in both structure and use.

The next chapter provides the details of the coding scheme that we used for this study including explanations of each coding category. Following that are the ten language particular chapters. Taken as a whole this issue illustrates how a combination of qualitative and quantitative methodologies can yield an overview of patterns in social interaction that is both interactionally sensitive and adequate for systematic comparative analyses. Such comparative analyses are necessary, not only in order to determine what is universal and what is culture-specific in human social interaction, but to understand what drives the diversity and what constrains it.

\section{References}

Atkinson, J.M., Drew, P., 1979. Order in Court: The Organisation of Verbal Interaction in Judicial Settings. Macmillan, London.

Bales, Robert Freed, 1950. Interaction Process Analysis: A Method for the Study of Small Groups. Addison-Wesley, Reading, MA.

Boyd, Elizabeth A., Heritage, John, 2006. Taking the patient's medical history: questioning during comprehensive history-taking. In: Heritage, J., Maynard, D.

(Eds.), Communication in Medical Care: Interactions between Primary Care Physicians and Patients. Cambridge University Press, Cambridge, England, pp. 151-184.

Brown, Penelope, 1998. Conversational structure and language acquisition: the role of repetition in Tzeltal adult and child speech. Journal of Linguistic Anthropology 8, 197-221.

Clayman, Steven, 2002. Sequence and solidarity. In: Lawler, E.J., Thye, S.R. (Eds.), Advances in Group Processes: Group Cohesion, Trust and Solidarity. Elsevier Science, Oxford, pp. 229-253.

Clayman, Steven, Heritage, John, 2002. The News Interview: Journalists and Public Figures on the Air. Cambridge University Press, Cambridge, England.

Clayman, Steven E., Elliott, Marc N., Heritage, John, McDonald, Laurie, 2006. Historical trends in questioning presidents 1953-2000. Presidential Studies Quarterly 36, 561-583.

Clayman, Steven E., Heritage, J., Elliott, M., McDonald, L., 2007. When does the watchdog bark? Conditions of aggressive questioning in presidential news conferences. American Sociological Review 72, 23-41.

Couper-Kuhlen, Elizabeth. Some truths and untruths about prosody in English question and answer sequences. In: de Ruiter, J.P. (Ed.), Questions: Formal, Functional and Interactional Perspectives. Cambridge University Press, Cambridge, in press.

Curl, Traci S., Drew, Paul, 2008. Contingency and action: a comparison of two forms of requesting. Research on Language and Social Interaction 41, 129-153.

Enfield, N.J., Levinson, Stephen C., 2006. Introduction: human sociality as a new interdisciplinary field. In: Enfield, N.J., Levinson, S.C. (Eds.), Roots of Human Sociality. Berg, Oxford, England, pp. 1-35.

Evans, Nicholas, Levinson, Stephen C., 2009. The myth of language universals: language diversity and its importance for cognitive science. Behavioral and Brain Sciences 32, 429-492.

Freed, Alice F., 1994. The form and function of questions in informal dyadic conversation. Journal of Pragmatics 21, 621-644.

Geluykens, Ronald, 1988. On the myth of rising intonation in polar questions. Journal of Pragmatics 12, $483-494$.

Goody, E., 1978. Questions and Politeness: Strategies in Social Interaction. Cambridge University Press, Cambridge.

Heritage, John, 1984. Garfinkel and Ethnomethodology. Polity Press, Cambridge.

Heritage, John, Raymond, Geoff, 2005. The terms of agreement: indexing epistemic authority and subordination in assessment sequences. Social Psychology Quarterly 68, 15-38.

Heritage, John, Raymond, Geoffrey. Navigating epistemic landscapes: acquiesence, agency and resistance in responses to polar questions. In: de Ruiter, J.P. (Ed.), Questions: Formal, Functional and Interactional Perspectives. Cambridge University Press, Cambridge, in press.

Heritage, John, Roth, Andrew, 1995. Grammar and institution: questions and questioning in the broadcast news interview. Research on Language and Social Interaction 28, 1-60.

Hymes, Dell, 1962. The ethnography of speaking. In: Gladwin, T., Sturtevant, W.C. (Eds.), Anthropology and Human Behavior. Anthropological Society of Washington, Washington DC, (Reprinted in J.A. Fishman, Readings in the Sociology of Language. Mouton, The Hague, 1968 , pp. 99-138), pp. 13-53. Jones, Bob Morris, 1999. The Welsh Answering System. Mouton de Gruyter, New York.

Kendon, Adam, 2002. Some uses of the head shake. Gesture 2, 147-182. 
Kita, Sotaro, Ide, Sachiko, 2007. Special Issue on nodding, aizuchi and final particles in Japanese conversation. Journal of Pragmatics 39.7, 1239-1332. Labov, William, Fanshel, David, 1977. Therapeutic Discourse: Psychotherapy as Conversation. Academic Press, New York.

Levinson, Stephen C, 1983. Pragmatics. Cambridge University Press, Cambridge.

Levinson, Stephen C., 2000. Presumptive Meanings: The Theory of Generalized Conversational Implicature. The MIT Press, Cambridge, Massachussetts.

Levinson, Stephen C., Brown, Penelope, 2004. Comparative Feedback: Cultural Shaping of Response Systems in Interaction, Feedback in Interaction. Max Planck Institute for Psycholinguistics, Nijmegen, The Netherlands.

Mangione-Smith, Rita, Elliott, Marc N., Stivers, Tanya, McDonald, Laurie L., Heritage, John, 2006. Ruling out the need for antibiotics: are we sending the right message? Archives of Pediatric and Adolescent Medicine 160, 945-952.

Maynard, Senko K., 1987. Interactional functions of a nonverbal sign: head movements in Japanese dyadic casual conversation. Journal of Pragmatics 11, 589-606.

Mehan, Hugh, 1985. The structure of classroom discourse. In: Dijk, T.A. (Ed.), Handbook of Discourse Analysis, vol. 3. Academic Press, New York, pp. 120131.

Pomerantz, Anita, 1984. Agreeing and disagreeing with assessments: some features of preferred/dispreferred turn shapes. In: Atkinson, J.M., Heritage, J. (Eds.), Structures of Social Action: Studies in Conversation Analysis. Cambridge University Press, Cambridge, pp. 57-101.

Raymond, Geoffrey, 2003. Grammar and social organization: yes/no interrogatives and the structure of responding. American Sociological Review 68, 939967.

Rossano, Federico, Brown, Penelope, Levinson, Stephen C., 2009. Gaze, questioning and culture. In: Sidnell, J. (Ed.), Conversation Analysis: Comparative Perspectives. Cambridge University Press, Cambridge, pp. 187-249.

Sacks, Harvey, 1987. On the preferences for agreement and contiguity in sequences in conversation. In: Button, G., Lee, J.R.E. (Eds.), Talk and Social Organisation. Multilingual Matters, Clevedon, England, pp. 54-69.

Sacks, Harvey, Schegloff, Emanuel A., Jefferson, Gail, 1974. A simplest systematics for the organization of turn-taking for conversation. Language 50, 696735.

Schegloff, Emanuel A., 1968. Sequencing in conversational openings. American Anthropologist 70, 1075-1095.

Schegloff, Emanuel A., Jefferson, Gail, Sacks, Harvey, 1977. The preference for self-correction in the organization of repair in conversation. Language 53, 361382.

Searle, John R., 1969. Speech Acts: An Essay in the Philosophy of Language. Cambridge University Press, Cambridge.

Steensig, Jakob, Drew, Paul, 2008. Questioning. Discourse Studies 10, 5-133.

Stenström, Anna-Brita, 1984. Questions and Responses in English Conversation. CWK Gleerup, Malmö, Sweden.

Stiles, W.B., 1989. Evaluating medical interview process components: null correlations with outcomes may be misleading. Medical Care 27, 212-220.

Stivers, Tanya. Morality and question design: ‘Of course' as contesting a presupposition of askability. In: Stivers, T., Mondada, L., Steensig, J. (Eds.), The Morality of Knowledge in Conversation. Cambridge University Press, Cambridge, forthcoming.

Stivers, Tanya, Enfield, N.J., Brown, Penelope, Englert, Christina, Hayashi, Makoto, Heinemann, Trine, Hoymann, Gertie, Rossano, Federico, de Ruiter, J.P., Yoon, Kyung-Eun, Levinson, Stephen C., 2009. Universality and cultural specificity in turn-taking in conversation. Proceedings of the National Academy of Science 106, 10587-10592.

Stivers, Tanya, Enfield, N.J., Levinson, Stephen C., February, 2010. Answering polar questions in social interaction. In: Workshop on Questions in Conversation (a Cross-Linguistic Project). University of York.

Stivers, Tanya, Hayashi, Makoto, 2010. Transformative Answers: One Way to Resist a Question's Constraints. Language in Society 39, 1-38.

Stivers, Tanya, Mangione-Smith, Rita, Elliott, Marc N., McDonald, Laurie, Heritage, John, 2003. Why do physicians think parents expect antibiotics? What parents report vs what physicians perceive. The Journal of Family Practice 52, 140-148.

Stivers, Tanya, Robinson, J.D., 2006. A preference for progressivity in interaction. Language in Society 35, 367-392.

Tuckett, D., Boulton, M., Olson, C., Williams, A., 1985. Meetings between Experts: An Approach to Sharing Ideas in Medical Consultations. Tavistock, London. Weber, Elizabeth, 1993. Varieties of Questions in English Conversation. John Benjamins, Amsterdam.

\author{
N.J. Enfield ${ }^{\mathrm{a}, \mathrm{b}, \mathrm{c} *}$ \\ aMax Planck Institute for Psycholinguistics, Wundtlaan 1, 6525XD Nijmegen, The Netherlands \\ ${ }^{\mathrm{b}}$ Radboud University Nijmegen, PB 9102, 6500 HC, The Netherlands \\ 'Donders Institute for Brain, Cognition, and Behaviour, PB 9102, 6500 HC, The Netherlands
} Stephen C. Levinson Max Planck Institute for Psycholinguistics, Wundtlaan 1, 6525XD Nijmegen, The Netherlands

*Corresponding author at: Max Planck Institute for Psycholinguistics, Wundtlaan 1, 6525XD Nijmegen, The Netherlands E-mail addresses: Nick.Enfield@mpi.nl (N.J. Enfield)

Tanya.Stivers@mpi.nl (T. Stivers) Stephen.Levinson@mpi.nl (S. Levinson)

${ }^{1}$ Permanent address: University of California, Los Angeles, 264 Haines Hall, 375 Portola Plaza, Los Angeles, CA 90095-1551, USA.

Received 29 March 2010 